\title{
Hot Pockets: The Adverse Admixture of Obesity and Diverticulitis
}

\author{
Vikram Rangan ${ }^{1} \cdot$ Judy Nee ${ }^{1}$ \\ Published online: 7 May 2020 \\ (c) Springer Science+Business Media, LLC, part of Springer Nature 2020
}

Diverticulosis is a common condition in the USA, affecting from 5 to $45 \%$ of the population in Western countries. Previously identified risk factors for diverticulosis (as well as diverticulitis) included modifiable lifestyle components such as diet (increased red meat intake, decreased fiber intake) as well as increasing age, smoking, alcohol use, and lack of physical activity. While obesity, i.e., BMI $>30$ in the USA, has been associated with an increased risk of developing diverticulosis in small studies, a recent study evaluating its association with obesity demonstrated an increased risk among women but not men [1]. Thus far, the effect of obesity on diverticulitis has not previously been studied in a large population.

In this issue of Digestive Diseases and Sciences, Patel et al. [2] in a well-written, large, retrospective study utilizing data from the Nationwide Readmission Database from 2010 to 2014 analyzed data from nearly 900,000 individuals with an index admission for diverticulitis. They found that while total admissions for diverticulitis decreased between 2010 and 2014, admission among morbidly obese individuals increased by over $50 \%$. They also demonstrated that in comparison to those with $\mathrm{BMI}<30$, those with morbid obesity had an increased risk for mortality, ICU admission, emergent surgery, and need for multiple admissions for diverticulitis. Morbidly obese individuals with diverticulitis were also younger on average than their non-obese counterparts.

The younger age and increased disease severity in diverticulitis patients with morbid obesity raises the evident question of whether these individuals represent a unique disease phenotype. Indeed, one key question is whether the earlier onset of diverticulitis in those with morbid obesity is a result of the earlier onset of diverticulosis in these patients (possibly due to increased intra-abdominal pressure, and to differences in underlying bowel habits), or whether the underlying

Judy Nee

jnee@bidmc.harvard.edu

1 Division of Gastroenterology, Department of Medicine, Beth Israel Deaconess Medical Center, Boston, MA, USA chronic inflammatory state caused by morbid obesity [3] predisposes to the development of diverticulitis. Though antibiotics have traditionally been the mainstay of management, there is evolving support for the concept of diverticulitis as more of an inflammatory rather than an infectious condition. Indeed, a recent meta-analysis of those with acute uncomplicated diverticulitis showed that antibiotic use did not improve clinical outcomes [4]. With this evidence, the 2015 AGA consensus document on the management of acute diverticulitis recommended that antibiotics be used selectively rather than routinely for acute uncomplicated diverticulitis [5].

One potential area of criticism of this study is that visceral (rather than subcutaneous) fat content is associated with the severity of diverticulitis [6]; in this study, the authors acknowledge that BMI (as a surrogate for visceral fat) is an imperfect marker for obesity. Clinicians making a new diagnosis of diverticulitis must make decisions regarding whether a given patient requires antibiotic treatment and can be managed on an outpatient versus an inpatient basis. Although visceral fat assessment is not a commonly used tool, BMI is easily calculated from the weight and height. Therefore, given the increased complication rate in this population, the presence of obesity (BMI $>30$ with comorbidities) or super-obesity (BMI $>40$ ) may push clinicians toward routine antibiotic use and/or inpatient management of newly diagnosed diverticulitis. The authors acknowledge the comorbidities that track with morbid obesity; one of the strengths of this article is its utilization of multivariable and propensity score matching analysis that accounts for these numerous obesity-associated comorbidities such as hypertension, COPD, diabetes, and heart failure. By controlling for multiple comorbidities, this article does suggest that there is an intrinsic effect of morbid obesity on diverticulitis morbidity and mortality. Nonetheless, further investigation into the mechanisms linking morbid obesity and poorer outcomes is needed.

The article also highlights the contributions of surgery to the management of diverticulitis in the morbidly obese. For the general population, although surgery is one of few 
effective interventions for reducing but not eliminating the risk of future episodes of diverticulitis [7], recent guidelines, including the most recent AGA consensus from 2015, have moved away from advocating routine elective surgery in those with recurrent episodes of diverticulitis [5]. The presence of a more severe disease phenotype among the morbidly obese patients with diverticulitis studied in this article would seemingly argue for a lower threshold for elective surgical intervention in these individuals. Nevertheless, the article also demonstrates the high risk for surgical intervention in this specific population, as more frequent surgical complications as well as need for colostomy and open colectomy occur. The surgeries that were noted by this study took place during an admission (either initial or subsequent) for diverticulitis, and thus the inferior outcomes with surgery among morbidly obese patients could also have reflected the increased severity of the underlying diverticulitis among these morbidly obese patients. Future studies of the outcomes of elective surgery in morbidly obese patients outside of an acute diverticulitis flare would thus be important in informing how aggressive providers should be in advocating surgical management in this population.

Given the previously described U-shaped relationship between obesity (both body weight as well as body fat) and mortality, the authors excluded those with mild-moderate obesity (BMI 30-40 without morbid obesity-defining comorbidities), as well as those with $\mathrm{BMI}<19$, allowing for a more distinct comparator group in this study. Nonetheless, given the markedly increasing prevalence of not just morbid obesity but also of mild/moderate obesity in the USA, an important area of future study is the implication of less severe forms of obesity on diverticular disease. Specifically, does the increased severity risk exist on a continuum with increasing BMI, or is it only observed above a threshold BMI value? This has clinical implications not only for the management of an acute episode of diverticulitis in a super obese patient, but also for determining whether weight loss may be recommended to obese patients with an episode of diverticulitis. Indeed, a frequent question among patients admitted for a complication of diverticular disease is whether any intervention (lifestyle or pharmacologic) can decrease the risk of future complications. Thus far, beyond fiber supplementation [8], there are limited evidence-based interventions available. In this study, those with a history of bariatric surgery were excluded from analysis. One potential future area of exploration would be to assess diverticulitis severity in those with a history of bariatric surgery versus BMI-matched controls, in order to assess the effect of weight loss on diverticulitis severity.

Though many questions remain unanswered, this article advances clinical practice by demonstrating that morbid obesity is associated with increased diverticulitis severity, even after controlling for associated comorbidities. While there is not enough evidence in this article to support a standard treatment approach to morbidly obese patients with diverticulitis, it does clearly demonstrate that morbidly obese patients suffer from high rates of complications and also require greater rates of hospitalization and antibiotic utilization.

\section{References}

1. Peery AF, Keil A, Jicha K, Galanko JA, Sandler RS. Association of obesity with colonic diverticulosis in women. Clin Gastroenterol Hepatol. 2020;18:107-114.

2. Patel K, Krishna SG, Porter K, et al. Diverticulitis in morbidly obese adults: a rise in hospitalizations with worse outcomes according to national US data. Dig Dis Sci. (Epub ahead of print). https://doi.org/10.1007/s10620-019-06002-w.

3. Ellulu MS, Patimah I, Khaza'ai H, Rahmat A, Abed Y. Obesity and inflammation: the linking mechanism and the complications. Arch Med Sci. 2017;13:851-863.

4. Mocanu V, Dang JT, Switzer N, et al. The role of antibiotics in acute uncomplicated diverticulitis: a systematic review and metaanalysis. Am J Surg. 2018;216:604-609.

5. Stollman N, Smalley W, Hirano I, Institute Clinical Guidelines Committee AGA. American gastroenterological association institute guideline on the management of acute diverticulitis. Gastroenterology. 2015;149:1944-1949.

6. Docimo S, Lee Y, Chatani P, Rogers AM, Lacqua F. Visceral to subcutaneous fat ratio predicts acuity of diverticulitis. Surg Endosc. 2017;31:2808-2812.

7. Bolkenstein HE, Consten ECJ, van der Palen J, et al. Long-term outcome of surgery versus conservative management for recurrent and ongoing complaints after an episode of diverticulitis: 5-year follow-up results of a multicenter randomized controlled trial (DIRECT-Trial). Ann Surg. 2019;269:612-620.

8. Ma W, Nguyen LH, Song M, et al. Intake of dietary fiber, fruits, and vegetables and risk of diverticulitis. Am J Gastroenterol. 2019;114:1531-1538.

Publisher's Note Springer Nature remains neutral with regard to jurisdictional claims in published maps and institutional affiliations. 